\title{
Stable causality of Black Saturns
}

\section{Sebastian J. Szybka}

Astronomical Observatory, Jagellonian University, Orla 171, 30-244 Kraków, Poland

E-mail: sebastian.szybka@uj.edu.pl

ABSTRACT: We prove that the Black Saturns are stably causal on the closure of the domain of outer communications.

KeYwords: Black Holes, Differential and Algebraic Geometry

ARXIV EPRINT: 1102.3942 


\section{Contents}

1 Introduction 1

2 Stable causality 2

\section{Introduction}

The Black Saturn solution of Elvang and Figueras is a five dimensional black hole with disjoint event horizons with topology $\mathbb{R} \times S^{3}$ and $\mathbb{R} \times S^{1} \times S^{2}$ [1, 2]. It describes a spherical black hole ${ }^{1}$ surrounded by a black ring.

In this article, we address the question of causality violations in the Black Saturn solution. The absence of closed causal curves is one of the desired properties of a solution to the Einstein equations. Such property should be stable against small perturbations. Therefore, the closed causal curves are not allowed in any Lorentzian metric that is sufficiently near the original one. This leads to the notion of stable causality as introduced by Hawking [3]. Technically, a spacetime $\left(M, g_{\mu \nu}\right)$ is stably causal if and only if there exists a differentiable function $f$ on $M$ such that $\nabla^{\mu} f$ is a past directed timelike vector field $[3,4]$. The non-unique function $f$ may be interpreted as a cosmic time that increases along every causal curve.

The natural guess for the Black Saturn is that the generalized Weyl $t$ coordinate is a cosmic time in the domain of outer communications (d.o.c.). Under this hypothesis, the problem of stable causality of the d.o.c. was reduced in [1] to the study of the $g_{\psi \psi}$ component of the metric. Namely, stable causality will result from the following inequality in the generalized Weyl coordinates

$$
g(\nabla t, \nabla t)=g^{t t}=-\frac{g_{\psi \psi}}{G_{y}}<0,
$$

where $G_{y}$ is a non-negative function which is zero only on the axis of the rotation of the Killing field $\partial_{\psi}$. In other words, it is sufficient to show that $g_{\psi \psi}$ vanishes as fast as $G_{y}$ on the axis of the rotation of $\partial_{\psi}$ and that $g_{\psi \psi}>0$ in the remaining part of the d.o.c.

Numerical evidence for positivity of $g_{\psi \psi}$ in the plane of the ring (as defined in [2, section 3.8]) was already signalled in the original paper of Elvang and Figueras [2]. This numerical evidence was extended in [1] to the part of spacetime covered by the generalized Weyl coordinates away from points where $\partial_{\psi}$ vanishes. Before our work, all analytical proofs of stable causality were restricted to the situation when the Komar angular momentum of the spherical component of the horizon is equal to zero: under this restriction, the positivity of $g_{\psi \psi}$ in the plane of the ring was established in [2], and stable causality of the d.o.c. was shown in [1].

\footnotetext{
${ }^{1}$ The adjective "spherical" refers to the topology of the horizon.
} 
In this article, we prove that the d.o.c. of the general Black Saturn solution is stably causal. In addition, we show that the event horizons may be included to the domain of stable causality, hence the Black Saturns are stably causal on the closure of the d.o.c. ${ }^{2}$

The calculations presented here ${ }^{3}$ involve manipulations of huge algebraic expressions and were done with Mathematica. We use the same notation and definitions of auxiliary functions as in [1].

\section{Stable causality}

In the generalized Weyl coordinates $(t, \psi, \varphi, \rho, z)$ the d.o.c. corresponds to

$$
\{\rho>0\} \cup\left\{\rho=0, z \notin\left[a_{5}, a_{4}\right] \cup\left[a_{3}, a_{2}\right]\right\},
$$

where $a_{i=1, \ldots, 5}$ are parameters. Since $g_{\psi \psi}=g_{\psi \psi}\left(\rho, z, a_{1}, \ldots, a_{5}\right)$, then we would like to prove stable causality for a union of the following sets in $\mathbb{R}^{7}$

$$
\begin{aligned}
\tilde{V}_{a} & =\left\{a_{1}<a_{5}<a_{4}<a_{3}<a_{2}\right\}, \\
\tilde{V}_{I} & =\{\rho>0\} \cap \tilde{V}_{a}, \\
\tilde{V}_{I I} & =\left\{\rho=0, z<a_{1}\right\} \cap \tilde{V}_{a}, \\
\tilde{V}_{I I I} & =\left\{\rho=0, a_{1} \leq z<a_{5}\right\} \cap \tilde{V}_{a}, \\
\tilde{V}_{I V} & =\left\{\rho=0, a_{4}<z<a_{3}\right\} \cap \tilde{V}_{a}, \\
\tilde{V}_{\psi} & =\left\{\rho=0, a_{2}<z\right\} \cap \tilde{V}_{a} .
\end{aligned}
$$

Hence, we have $\tilde{V}_{\text {d.o.c. }}=\tilde{V}_{I} \cup \tilde{V}_{I I} \cup \tilde{V}_{I I I} \cup \tilde{V}_{I V} \cup \tilde{V}_{\psi}$. We are interested in non-degenerate solutions, so the parameters are restricted to $\tilde{V}_{a}$. The "plane of the ring" corresponds to $\tilde{V}_{I I} \cup \tilde{V}_{I I I} \cup \tilde{V}_{I V}$, while $\tilde{V}_{\psi}$ is the intersection of the rotation axis of $\partial_{\psi}$ with the d.o.c. The event horizons of the black ring and the spherical component coincide with $(\{\rho=$ $\left.0\} \backslash \tilde{V}_{\text {d.o.c. }}\right) \cap \tilde{V}_{a}$. We would like to show that $g_{\psi \psi}>0$ on $\tilde{V}_{I} \cup \tilde{V}_{I I} \cup \tilde{V}_{I I I} \cup \tilde{V}_{I V}$. Moreover, since $G_{y}$ vanishes as $\rho^{2}$ on the axis of $\partial_{\psi}$ [1], then it is necessary to check that

$$
\lim _{\rho \rightarrow 0^{+}} \frac{g_{\psi \psi}}{\rho^{2}}>0
$$

on $\tilde{V}_{\psi}$.

It turns out to be convenient to view $g_{\psi \psi}$ as a function of $\rho, \mu_{1}, \ldots, \mu_{5}$, where $\mu_{i}=$ $\sqrt{\rho^{2}+\left(z-a_{i}\right)^{2}}-\left(z-a_{i}\right)$. In this parametrization, the translational symmetry of $z, a_{i}$ is explicit. The analogues of the sets ${ }^{4}(2.1)$ are now in $\mathbb{R}^{6}$

$$
\begin{aligned}
& V_{\mu}=\left\{\rho \leq \mu_{1} \leq \mu_{5} \leq \mu_{4} \leq \mu_{3} \leq \mu_{2}\right\}, \\
& V_{I}=\{\rho>0\} \cap V_{\mu},
\end{aligned}
$$

\footnotetext{
${ }^{2}$ In fact, our proof remains valid for a family of the Black Saturn solutions with conical singularities on the axes of the periodic Killing fields $\partial_{\psi}, \partial_{\varphi}$.

${ }^{3}$ The Mathematica code is available at http://th.if.uj.edu.pl/ szybka/BScausality.

${ }^{4}$ These sets are not equivalent to $(2.1)$ because $V_{4} \subset V_{I V}, V_{2} \subset V_{\psi}$, where $V_{4}, V_{2}$ correspond to the sets that are defined in the old parametrization as $\left\{\rho=0, z=a_{4}\right\} \cap \tilde{V}_{a},\left\{\rho=0, z=a_{2}\right\} \cap \tilde{V}_{a}$, respectively.
} 


$$
\begin{aligned}
V_{I I} & =\left\{\rho=0, \mu_{1}>0\right\} \cap V_{\mu}, \\
V_{I I I} & =\left\{\rho=0, \mu_{1}=0, \mu_{5}>0\right\} \cap V_{\mu}, \\
V_{I V} & =\left\{\rho=0, \mu_{4}=0, \mu_{3}>0\right\} \cap V_{\mu}, \\
V_{\psi} & =\left\{\rho=0, \mu_{2}=0\right\} \cap V_{\mu},
\end{aligned}
$$

and, of course, $V_{\text {d.o.c. }} \subset V_{I} \cup V_{I I} \cup V_{I I I} \cup V_{I V} \cup V_{\psi}$. It follows from the definition of $\mu_{i}$ that if $\mu_{i}=\mu_{j}$ for $i \neq j$, then $\rho=\mu_{i}=\mu_{j}=0$. This parametrization turns out to be more helpful in completing the proof.

The numerator and the denominator of $g_{\psi \psi}$, when written as polynomials in $\rho, \mu_{i}$, and some $c_{1}, c_{2}, q$ contain tens of thousands monomials $[1,2]$. One may check with a direct Mathematica calculation that some non-trivial factors from the numerator and the denominator cancel and the original form of $g_{\psi \psi}$ may be simplified to

$$
g_{\psi \psi}=\frac{\mu_{4} \mu_{5} A^{2}-\mu_{3} B^{2}}{\mu_{1} \mu_{4} H_{x} F},
$$

where

$$
\begin{aligned}
& A=\left(\mu_{2} p_{1}\left(\mu_{5} p_{2}+c_{2} q \mu_{1} \mu_{3} p_{3}\right)+c_{1} \mu_{3} p_{4}\left(-q \rho^{2} p_{5}+c_{2} \mu_{1} \mu_{4} p_{6}\right)\right), \\
& B=\left(\mu_{5} p_{1}\left(q \mu_{1} p_{2}-c_{2} \mu_{4} \rho^{2} p_{3}\right)+c_{1} \mu_{2} \mu_{4} p_{4}\left(\mu_{1} \mu_{5} p_{5}+c_{2} q \mu_{3} p_{6}\right)\right), \\
& p_{1}=\left(\mu_{3}-\mu_{1}\right)\left(\mu_{1} \mu_{4}+\rho^{2}\right) \\
& p_{2}=\left(\mu_{2}-\mu_{4}\right)\left(\mu_{1} \mu_{2}+\rho^{2}\right)\left(\mu_{2} \mu_{3}+\rho^{2}\right) \\
& p_{3}=\left(\mu_{2}-\mu_{1}\right)\left(\mu_{2} \mu_{5}+\rho^{2}\right) \\
& p_{4}=\mu_{1}\left(\mu_{5}-\mu_{1}\right) \\
& p_{5}=\left(\mu_{2}-\mu_{1}\right)\left(\mu_{2}-\mu_{4}\right)\left(\mu_{2} \mu_{3}+\rho^{2}\right), \\
& p_{6}=\left(\mu_{1} \mu_{2}+\rho^{2}\right)\left(\mu_{2} \mu_{5}+\rho^{2}\right)
\end{aligned}
$$

and $p_{i} \geq 0$. The functions $H_{x}, F$ are non-negative and they were defined in [2]. It follows from the analysis in [1] that zeros of $H_{x} F$ exist only for $\rho=0$ and they cancel with the zeros of the numerator of $g_{\psi \psi}$.

The parameters $c_{1}, c_{2}, q$ depend only on $a_{i}$ and do not depend on $\rho, z$. However, if one assumes that $\rho, \mu_{i}$ are independent variables, then $c_{1}, c_{2}, q$ are finite continuous functions of $\rho, \mu_{i}$ and are given by

$$
\begin{aligned}
c_{1}^{2} & =\frac{\left(\mu_{3}-\mu_{1}\right)\left(\mu_{4}-\mu_{1}\right) \mu_{5}\left(\mu_{1} \mu_{3}+\rho^{2}\right)\left(\mu_{1} \mu_{4}+\rho^{2}\right)}{\mu_{1} \mu_{3} \mu_{4}\left(\mu_{5}-\mu_{1}\right)\left(\mu_{1} \mu_{5}+\rho^{2}\right)}, \\
q & =\frac{c_{1} c_{2} \mu_{4}\left(\mu_{2}-\mu_{1}\right)\left(\mu_{1} \mu_{2}+\rho^{2}\right)}{c_{1} \mu_{1}\left(\mu_{2}-\mu_{4}\right)\left(\mu_{2} \mu_{4}+\rho^{2}\right)+c_{2} \mu_{2}\left(\mu_{4}-\mu_{1}\right)\left(\mu_{1} \mu_{4}+\rho^{2}\right)},
\end{aligned}
$$

where we imposed (2.5) on $c_{1}$ in the formula for $q$. The formula for $c_{2}$ in terms of $\rho, \mu_{i}$ is to long to be usefully cited here. It may be derived from equations $(4.2),(5.1)$ in [1], but it is not necessary for our calculations.

The formulas (2.5), (2.6) are explicitly valid in $V_{I} \cup V_{I I}$. They are also valid in the remaining part of the d.o.c. provided the limit $\rho \rightarrow 0^{+}$is carefully taken. If $\mu_{i}(\rho=0)=0$, then taking this limit should be preceded by the substitution $\mu_{i} \rightarrow \rho^{2} \hat{\mu}_{i}$, where $\hat{\mu}_{i}>0$ [1].

The simplification (2.3) is a significant one. Even if one does not substitute formulas for $c_{1}, c_{2}, q$, the original expression for $g_{\psi \psi}$ written as a rational function contained 106995 monomials. This number was reduced to 2344 in the simplified formula. 
In order to present the proof in a concise form, we introduce the following operators. Let $N, D$ denote operators acting on a rational functions that return polynomials: a numerator or a denominator, respectively. The result is not unique and the action of $N, D$ is given only up to an overall factor. However, this non-uniqueness is not important for our problem. We also define substitution operators $S_{q}$, respectively $S_{c_{1}^{2}}$, that return the rational function which is obtained after $q$, respectively $c_{1}^{2}$, has been substituted in the original expression using (2.6), respectively (2.5). One should note that $S_{q}$ and $S_{c_{1}^{2}}$ do not commute with $N$ and $D$ in general. The formula for $c_{2}$, in contrast to the formula for $c_{1}$, contains a square root that cannot be eliminated by taking $c_{2}^{2}$. We prefer to preserve the polynomial form of the evaluated expressions, hence we will substitute only $c_{1}^{2}$ and avoid substituting $c_{2}$.

The calculations described below were done with Mathematica. We present them here in a brief form. It follows from the smoothness of the Black Saturns [1] that the expressions evaluated below are regular.

We start with the analysis of $g_{\psi \psi}$ on $V_{I}(\rho>0) .{ }^{5}$ The denominator of $g_{\psi \psi}$ is given by $\mu_{1} \mu_{4} H_{x} F$ and it is a positive function on $V_{I}$. The numerator of $g_{\psi \psi}$ is equal to $\Xi_{+} \Xi_{-}$, where

$$
\Xi_{ \pm}=\sqrt{\mu_{4} \mu_{5}} A \pm \sqrt{\mu_{3}} B .
$$

If $\Xi_{+} \Xi_{-}>0$, then $g_{\psi \psi}>0$ on $V_{I}$, as desired. Firstly, we check at a random point ${ }^{6} P \in V_{I}$ that $\left.\Xi_{ \pm}\right|_{P}>0$. Since both $\Xi_{+}$and $\Xi_{-}$are continuous in $\rho, \mu_{i}$, then it is sufficient to show that they cannot vanish. The functions $\Xi_{ \pm}$are linear in $q$, as may be seen from (2.4), (2.7). We substitute $q$ into $\Xi_{ \pm}$and examine the numerators of the resulting expressions. We would like to show that none of them $\left(N S_{q} \Xi_{ \pm}\right)$has zeros. By inspection, we find that $N S_{q} \Xi_{ \pm}$ are quadratic in $c_{2}$. Since $c_{2}$ is real, a negative discriminant of $N S_{q} \Xi_{ \pm}$with respect to $c_{2}$ would imply that none of the equations $N S_{q} \Xi_{ \pm}=0$ has a solution. We calculate these discriminants $\Delta_{ \pm}$and they turn out to be fourth order in $c_{1}$. Next, we substitute $c_{1}^{2}$ into $\Delta_{ \pm}$ using (2.5) and taking $c_{1}^{4}=\left(c_{1}^{2}\right)^{2}, c_{1}^{3}=c_{1} c_{1}^{2}$. With a help of Mathematica we have derived

$$
S_{c_{1}^{2}} \Delta_{ \pm}=w_{ \pm} \frac{\mu_{1} \mu_{2}^{2} \mu_{4} \mu_{5}^{5 / 2}\left(\mu_{1}-\mu_{2}\right)^{2}\left(\mu_{3}-\mu_{1}\right)\left(\mu_{4}-\mu_{1}\right)\left(\mu_{2}-\mu_{4}\right)^{2}\left(\mu_{1} \mu_{2}+\rho^{2}\right)^{2}\left(\mu_{1} \mu_{4}+\rho^{2}\right)^{2}}{\left(\mu_{5}-\mu_{1}\right)\left(\mu_{1} \mu_{5}+\rho^{2}\right)^{2}} .
$$

The factors multiplying $w_{ \pm}$are strictly positive, and $w_{ \pm}$are complicated polynomials in $\rho$, $\sqrt{\mu_{i}}$. These polynomials are linear in $c_{1}$ (with non-vanishing coefficients in front of $c_{1}$ as it will follow from our further analysis). We check at a random point $P^{\prime} \in V_{I}$ that $\left.w_{ \pm}\right|_{P^{\prime}}<0$, hence if $w_{ \pm}$have no zeros, then $\Delta_{ \pm}<0$. Let $c_{1}^{ \pm}$be solutions to the equations $w_{ \pm}=0$. A MATHEMATICA calculation reveals that $c_{1}^{+}=-c_{1}^{-}$. We set $U=\left(c_{1}^{ \pm}\right)^{2}-S_{c_{1}^{2}} c_{1}^{2}$ and calculate

$$
U=\frac{\mu_{5}\left(\mu_{1}^{2}+\rho^{2}\right)^{2}}{4 \mu_{1}^{2} \mu_{3} \mu_{4}\left(\mu_{1}-\mu_{5}\right)^{2}\left(\mu_{1} \mu_{5}+\rho^{2}\right)^{2}} \frac{\hat{U}}{\tilde{U}}
$$

where $\hat{U}, \tilde{U}$ are complicated polynomials ${ }^{7}$ in $\rho, \mu_{i}$ with signs unknown so far. The coefficient in front of $\hat{U} / \tilde{U}$ is strictly positive. Now, we succeeded in making the signs of $\hat{U}, \tilde{U}$

\footnotetext{
${ }^{5} V_{I}=\{\rho>0\} \cap V_{\mu}$ as indicated in (2.2) , but for the sake of brevity we will remind only first part of the definitions.

${ }^{6}$ We impose the equation satisfied by $c_{2}$ in such checks, but this is actually not necessary.

${ }^{7}$ The polynomial $\tilde{U}$ is a full square.
} 
explicit by writing them in terms of the new positive functions

$$
\Delta_{51}=\mu_{5}-\mu_{1}, \quad \Delta_{45}=\mu_{4}-\mu_{5}, \quad \Delta_{34}=\mu_{3}-\mu_{4}, \quad \Delta_{23}=\mu_{2}-\mu_{3} .
$$

The coefficients in $\hat{U}, \tilde{U}$ in front of $\rho, \mu_{1}, \Delta_{i j}$ turn out ${ }^{8}$ to be positive and belong to

$$
[9,13705432344] \cap \mathbb{Z}, \quad[1,137075730] \cap \mathbb{Z},
$$

respectively. Since $\rho, \mu_{1}, \Delta_{i j}$ are strictly positive, then it follows that $\hat{U}, \tilde{U}$ are strictly positive and the equation $U=0$ does not have solutions. Therefore, $c_{1}^{ \pm} \neq S_{c_{1}^{2}} c_{1}$ and the polynomials $w_{ \pm}$cannot vanish. This means that the discriminants of $N S_{q} \Xi_{ \pm}$in respect to $c_{2}$ are negative $\left(\Delta_{ \pm}<0\right)$ and there are no real $c_{2}$ that would satisfy any of the equations $\Xi_{ \pm}=0$. Finally, this implies that $g_{\psi \psi}>0$ on $V_{I}$ (for $\rho>0$ ), as desired. To complete the proof it is sufficient to repeat the analysis above in the remaining part of the d.o.c.

For $\rho=0$ the denominator of $g_{\psi \psi}$ (given by $\mu_{1} \mu_{4} H_{x} F$ ) is not strictly positive any more. However, it follows from the Black Saturns' smoothness [1] that whenever the denominator of $g_{\psi \psi}$ vanishes the numerator of $g_{\psi \psi}$ (equal to $\Xi_{+} \Xi_{-}$) vanishes as well and the $\operatorname{limit}_{\rho \rightarrow 0^{+}} g_{\psi \psi}$ is finite. Moreover, the continuity of $g_{\psi \psi}$ implies that this limit is non-negative, possibly zero.

On $V_{I I}\left(\rho=0, \mu_{1}>0\right)$ the argument proceeds along the same lines as for $V_{I}$. Intermediate expressions have different form, but the reasoning is analogous. We have found

$$
U=\frac{\mu_{1}^{2}}{4 \mu_{3} \mu_{4} \mu_{5}\left(\mu_{1}-\mu_{5}\right)^{2}} \frac{\hat{U}}{\tilde{U}} .
$$

The coefficients in the polynomials $\hat{U}, \tilde{U}$ in front of $\mu_{1}, \Delta_{i j}$ range in

$$
[9,7882] \cap \mathbb{Z}, \quad[9,184] \cap \mathbb{Z},
$$

respectively. Hence, $U>0$ and none of the expressions $N S_{q} \Xi_{ \pm}$vanishes, and $g_{\psi \psi}>0$ on $V_{I I}$.

In order to study $g_{\psi \psi}$ on $V_{I I I}\left(\rho=\mu_{1}=0, \mu_{5}>0\right)$ we substitute $\mu_{1}=\rho^{2} \hat{\mu}_{1}$ into $\Xi_{ \pm}$ (the numerator of $g_{\psi \psi}$ is given by $\Xi_{+} \Xi_{-}$). It turns out that $\rho^{4}$ factors out in each term $\Xi_{ \pm}$. On the other hand, $\rho^{8}$ factors in $\mu_{1} \mu_{4} H_{x} F$ (the denominator of $g_{\psi \psi}$ ). We set

$$
\breve{\Xi}_{ \pm}=\lim _{\rho \rightarrow 0^{+}} \frac{\Xi_{ \pm}}{\rho^{4}}
$$

and repeat the proof for $\breve{\Xi}_{ \pm}$as in the case $\rho>0$. We have

$$
U=\frac{1}{4 \hat{\mu}_{1}^{2} \mu_{3} \mu_{4} \mu_{5}\left(1+\hat{\mu}_{1} \mu_{5}\right)^{2}} \frac{\hat{U}}{\tilde{U}}
$$

Finally, the coefficients in the polynomials $\hat{U}, \tilde{U}$ in front of the strictly positive functions $\hat{\mu}_{1}, \Delta_{i j}$ are again greater than zero and in

$$
[9,8714] \cap \mathbb{Z}, \quad[9,184] \cap \mathbb{Z},
$$

\footnotetext{
${ }^{8}$ We have $\mu_{1}>\rho$, but $\Delta_{i j}$ do not have to form monotonically increasing sequence like $\mu_{i}$.
} 
respectively. Therefore, $g_{\psi \psi}>0$ on $V_{I I I}$, as expected.

We continue our analysis on $V_{I V}\left(\rho=\mu_{4}=0, \mu_{3}>0\right)$. Here, $\mu_{1}=\rho^{2} \hat{\mu}_{1}, \mu_{5}=\rho^{2} \hat{\mu}_{5}$, $\mu_{4}=\rho^{2} \hat{\mu}_{4}$. We note that $0<\hat{\mu}_{1}<\hat{\mu}_{5}<\hat{\mu}_{4}$. The calculations are similar to the calculations for $V_{I I I}$. A factor $\rho^{16}$ appears both in the numerator and the denominator of $g_{\psi \psi}$, thus we define

$$
\breve{\Xi}_{ \pm}=\lim _{\rho \rightarrow 0^{+}} \frac{\Xi_{ \pm}}{\rho^{8}}
$$

and apply our standard analysis to $\breve{\Xi}_{ \pm}$. The formula for $U$ is

$$
U=\frac{\hat{\mu}_{5}}{4 \hat{\mu}_{1}^{2} \hat{\mu}_{4} \mu_{2}^{2} \mu_{3}^{3}\left(\hat{\mu}_{1}-\hat{\mu}_{5}\right)^{2}\left(1+\hat{\mu}_{1} \mu_{2}\right)^{2}} \hat{U} .
$$

In the final step we introduce $\Delta_{51}=\rho^{2} \hat{\Delta}_{51}, \Delta_{45}=\rho^{2} \hat{\Delta}_{45}$ and the coefficients in $\hat{U}$ in front of strictly positive functions $\hat{\mu}_{1}, \hat{\Delta}_{51}, \hat{\Delta}_{45}, \Delta_{34}, \Delta_{23}$ are greater than zero and belong to $[9,5852] \cap \mathbb{Z}$. Hence, $g_{\psi \psi}>0$ on $V_{I V}$, as desired.

In the case of the rotation axis of the periodic Killing field $\partial_{\psi}$ (the set $V_{\psi}$, where $\rho=\mu_{i}=0$ ), the analysis is slightly more involved. We substitute $\mu_{i}=\rho^{2} \hat{\mu}_{i}$ into $\Xi_{ \pm}$. Then, we have verified that $\Xi_{ \pm} \sim \rho^{15}$ for generic $c_{1}$ and $q$. However, a MathematicA calculation reveals that for our choice of $c_{1}^{2}$ and $q$ (the equations (2.5), (2.6)) the leading terms vanish and we have at least $\Xi_{ \pm} \sim \rho^{16}$. Therefore, we drop the leading terms in $\Xi_{ \pm}$and analyse the remaining higher order terms. We denote them with $\Xi_{ \pm}^{\prime}$. Next, we set

$$
\breve{\Xi}_{ \pm}=\lim _{\rho \rightarrow 0^{+}} \frac{\Xi_{ \pm}^{\prime}}{\rho^{16}}
$$

and apply our standard procedure to $\breve{\Xi}_{ \pm}$. We have found that

$$
S_{c_{1}^{2}} \Delta_{ \pm}=-\frac{4 \hat{\mu}_{1} \hat{\mu}_{2}^{2} \hat{\mu}_{4} \hat{\mu}_{5}^{3}\left(\hat{\mu}_{1}-\hat{\mu}_{2}\right)^{2}\left(\hat{\mu}_{3}-\hat{\mu}_{1}\right)^{3}\left(\hat{\mu}_{4}-\hat{\mu}_{1}\right)\left(\hat{\mu}_{2}-\hat{\mu}_{4}\right)^{2}}{\hat{\mu}_{5}-\hat{\mu}_{1}}
$$

which is strictly negative because $0<\hat{\mu}_{1}<\hat{\mu}_{5}<\hat{\mu}_{4}<\hat{\mu}_{3}<\hat{\mu}_{2}$. Thus, we have $\Xi_{+} \Xi_{-} \sim \rho^{32}$. The denominator of $g_{\psi \psi}$ (given by $\mu_{1} \mu_{4} H_{x} F$ ) behaves like $\rho^{30}$ and $g_{\psi \psi}$ vanishes like $\rho^{2}$. This, together with positivity of $g_{\psi \psi}$ for $\rho>0$ and continuity of $g_{\psi \psi}$, implies that

$$
\lim _{\rho \rightarrow 0^{+}} \frac{g_{\psi \psi}}{\rho^{2}}>0
$$

on the axis of the rotation of $\partial_{\psi}$ and the inequality (1.1) holds. It completes the proof of stable causality of the Black Saturns' d.o.c.

The generalized Weyl coordinates cover also the sets

$$
\begin{aligned}
& V_{b r}=\left\{\rho=0, \mu_{5}=0, \mu_{4}>0\right\} \cap V_{\mu}, \\
& V_{s b}=\left\{\rho=0, \mu_{3}=0, \mu_{2}>0\right\} \cap V_{\mu},
\end{aligned}
$$

which have been ignored in our analysis so far. The set $V_{B R}=V_{b r} \cup V_{4}$ corresponds to the event horizon of a black ring and the set $V_{S B}=V_{s b} \cup V_{2}$ corresponds to the event horizon 
of a spherical black hole. ${ }^{9}$ The closure of $V_{\text {d.o.c. }}$ is given by $V_{\text {d.o.c. }} \cup V_{B R} \cup V_{S B}$. Since $V_{4} \subset V_{I V}, V_{2} \subset V_{\psi}$, it remains to apply our analysis on $V_{b r}, V_{s b}$.

On $V_{b r}\left(\rho=\mu_{5}=0, \mu_{4}>0\right)$ we substitute $\mu_{1}=\rho^{2} \hat{\mu}_{1}, \mu_{5}=\rho^{2} \hat{\mu}_{5}$ into $\Xi_{ \pm}$. Then, it turns out that $\rho^{7}$ factors out of $\Xi_{ \pm}$and $\rho^{14}$ factors out of the denominator of $g_{\psi \psi}$ $\left(\mu_{1} \mu_{4} H_{x} F\right)$. We define

$$
\breve{\Xi}_{ \pm}=\lim _{\rho \rightarrow 0^{+}} \frac{\Xi_{ \pm}}{\rho^{7}}
$$

and calculate the discriminants $\Delta_{ \pm}$of $N S_{q} \Xi_{ \pm}$in respect to $c_{2}$. Next, we substitute $c_{1}^{2}$ into $\Delta_{ \pm}$and obtain

$$
S_{c_{1}^{2}} \Delta_{ \pm}=-w_{ \pm} \frac{\hat{\mu}_{1} \hat{\mu}_{5}^{3} \mu_{2}^{4} \mu_{3}^{4} \mu_{4}\left(1+\hat{\mu}_{1} \mu_{2}\right)^{2}\left(1+\hat{\mu}_{1} \mu_{4}\right)^{2}\left(\mu_{2}-\mu_{4}\right)^{2}}{\hat{\mu}_{5}-\hat{\mu}_{1}}
$$

where the coefficient behind $w_{ \pm}$is strictly greater than zero. This time $w_{ \pm}$are polynomials in $\rho, \hat{\mu}_{1}, \hat{\mu}_{5}, \mu_{4}, \mu_{3}, \mu_{2}$. They become explicitly positive if written in terms of $\hat{\mu}_{1}, \hat{\Delta}_{51}, \Delta_{45}$, $\Delta_{34}, \Delta_{23}$ with coefficients in the range $[3,16] \cap \mathbb{Z}$. Then, $S_{c_{1}^{2}} \Delta_{ \pm}<0$ and $g_{\psi \psi}>0$ on $V_{b r}$.

The analysis of positivity of $g_{\psi \psi}$ on $V_{s b}\left(\rho=\mu_{3}=0, \mu_{2}>0\right)$ mimics the calculations on $V_{b r}$. There are the following changes. We substitute $\mu_{1}=\rho^{2} \hat{\mu}_{1}, \mu_{5}=\rho^{2} \hat{\mu}_{5}, \mu_{4}=\rho^{2} \hat{\mu}_{4}$, $\mu_{3}=\rho^{3} \hat{\mu}_{4}$ into $\Xi_{ \pm}$. We set

$$
\breve{\Xi}_{ \pm}=\lim _{\rho \rightarrow 0^{+}} \frac{\Xi_{ \pm}}{\rho^{12}}
$$

and obtain

$$
S_{c_{1}^{2}} \Delta_{ \pm}=-\frac{4 \hat{\mu}_{1} \hat{\mu}_{4} \hat{\mu}_{5}^{3} \mu_{2}^{4}\left(1+\hat{\mu}_{1} \mu_{2}\right)^{2}\left(1+\hat{\mu}_{3} \mu_{2}\right)\left(1+\hat{\mu}_{4} \mu_{2}\right)\left(1+\hat{\mu}_{5} \mu_{2}\right)\left(\hat{\mu}_{3}-\hat{\mu}_{1}\right)^{3}\left(\hat{\mu}_{4}-\hat{\mu}_{1}\right)}{\hat{\mu}_{5}-\hat{\mu}_{1}}
$$

which is strictly negative. Therefore, $g_{\psi \psi}>0$ on $V_{s b}$.

In summary, our analysis applied to $V_{b r}, V_{s b}$ establishes that $g_{\psi \psi}>0$ there. The strict positivity of $g_{\psi \psi}$ remains valid on $V_{4} \subset V_{I V}, V_{2} \subset V_{\psi}$, as we know from the analysis of the sets $V_{I V}, V_{\psi}$. Therefore, $g_{\psi \psi}>0$ holds on both event horizons $V_{B R}, V_{S B}$. This implies that the inequality (1.1) is satisfied on the event horizons. They may be included to the Black Saturns' domain of stable causality and the Black Saturns are stably causal on the closure of the d.o.c.

We stress that we have not assigned a particular value to $c_{2}$ in our calculations so all results hold also for the Black Saturns with conical singularities on the axes of the rotation of the periodic Killing fields $\partial_{\psi}, \partial_{\varphi}$.

\section{Acknowledgments}

We are grateful to Piotr Chruściel and Michał Eckstein for useful discussions. Our calculations were carried out with MATHEMATiCA. This research was supported by the Foundation for Polish Science.

\footnotetext{
${ }^{9}$ The sets $V_{4}, V_{2}$ were defined in the footnote on page 3.
} 
Open Access. This article is distributed under the terms of the Creative Commons Attribution Noncommercial License which permits any noncommercial use, distribution, and reproduction in any medium, provided the original author(s) and source are credited.

\section{References}

[1] P.T. Chruściel, M. Eckstein and S.J. Szybka, On smoothness of Black Saturns, JHEP 11 (2010) 048 [arXiv: 1007.3668] [SPIRES].

[2] H. Elvang and P. Figueras, Black Saturn, JHEP 05 (2007) 050 [hep-th/0701035] [SPIRES].

[3] S. Hawking, The existence of cosmic time functions, Proc. Roy. Soc. Lond. A 308 (1969) 433 [SPIRES].

[4] S.W. Hawking and G.F.R. Ellis, The large-scale structure of space-time, Cambridge University Press, Cambridge U.K. (1973) [SPIRES]. 